\title{
Coulomb-enhanced resonance transmission of quantum SINIS junctions
}

\author{
N. B. Kopnin,,$^{1,2}$ Y. M. Galperin, ${ }^{3,4}$ and V. M. Vinokur ${ }^{5}$ \\ ${ }^{1}$ Low Temperature Laboratory, Aalto University, PO Box 15100, FI-00076 AALTO, Finland \\ ${ }^{2}$ L. D. Landau Institute for Theoretical Physics, 117940 Moscow, Russia \\ ${ }^{3}$ Department of Physics, University of Oslo, PO Box 1048 Blindern, 0316 Oslo, Norway \\ ${ }^{4}$ A. F. Ioffe Physico-Technical Institute of Russian Academy of Sciences, 194021 St. Petersburg, Russia \\ ${ }^{5}$ Argonne National Laboratory, 9700 S. Cass Ave., Argonne, IL 60439, USA
}

(Dated: November 18, 2018)

\begin{abstract}
Coherent charge transfer through a ballistic gated SINIS junction is mediated by the resonant tunneling via the Andreev states. Extra charge accommodated on the Andreev levels partially compensates the charge induced by the gate voltage preserving the electron wavelength and maintaining the resonance conditions in a broad range of gate voltages. As a result, the transparency of the junction as well as the supercurrent trough it can be substantially increased as compared to the zero-Coulomb case.
\end{abstract}

PACS numbers: $74.45 .+\mathrm{c}, 74.50 .+\mathrm{r}, 73.63 .-\mathrm{b}$

According to the classical picture of the Coulomb blockade in gated structures [1] the Coulomb interaction usually suppresses electronic transport through small conductors by introducing an additional energy barrier associated with the charging energy. In this Letter we show that in ballistic SINIS junctions (here S stands for a superconductor, $\mathrm{N}$ is a normal-metal island, and $\mathrm{I}$ is an insulator), the Coulomb interaction can rather stimulate the supercurrent than suppress it. The supercurrent through a junction containing a ballistic normal island is mediated by Andreev states with energies controlled by the effective transparency of the double-barrier structure which is determined by the wave length of electrons. The extra charge on the dot affects not only the energy but also the wave length of electrons. This makes the transmission extremely sensitive both to the gate voltage and to the charge on the Andreev states localized in the normal island. We show that charging of Andreev states can actually preserve the condition of resonance tunneling: the charge adjusts itself so as to compensate the deviation of the chemical potential caused by the change in the gate voltage thus increasing the transparency of the device for the supercurrent.

The system. - We consider the normal island in the form of a short single-mode ballistic conductor (wire) connected to bulk superconducting leads via lowtransparency contacts. This corresponds to the common experimental situation which is realized, in particular, in recent experiments on nanowires [2]. The length $d$ of a conductor is much less than the superconducting coherence length, $\xi$, i.e., $|\Delta| \ll \delta E$ where $\delta E \sim \hbar v_{F} / d$ is the level spacing in the double-barrier structure, $v_{F}$ is the velocity of electrons in a quantum conductor, and $|\Delta|$ is the superconducting gap in the leads. The short conductor (sometimes called an Andreev quantum dot) is weakly coupled to the superconducting leads and forms a low-proximity device. This setup is similar to that considered in Refs. 3] where the charging of Andreev levels was studied in the limit of small Coulomb interaction.

To characterize the efficiency of the Coulomb interaction in the SINIS we introduce the dimensionless parameter $\gamma=E_{C} / \delta E \sim e^{2} d / C \hbar v_{F}$, where $E_{C}=e^{2} / 2 C$ is the Coulomb energy of the island, $C$ being its capacitance. The maximum value of $\gamma$ is less than $e^{2} / \hbar v_{F} \varkappa$, with the island capacitance $C \sim \varkappa d \ln (d / a)$, where $a$ is the transverse dimension of the wire and $\varkappa$ is the effective dielectric constant in the presence of a substrate. For a typical value $v_{F} \sim 10^{8} \mathrm{~cm} / \mathrm{s}$ the corresponding $\gamma$ is small; thus the assumption $\gamma \ll 1$ is adequate for practical devices. The same parameter characterizes the Luttingerliquid effects [4], which thus can be neglected. However, in spite of the small value of $\gamma$, the Coulomb energy can still be either larger or smaller than the superconducting gap. To account for nonlinear back-action effects on the contact transparency associated with charging of the Andreev level in case of a considerable Coulomb energy $E_{C} \sim|\Delta|$, we adopt a mean-field model similar to that employed in Ref. [5] for normal junctions. In this respect, our model differs from those considering transport through quantum dots having a single fixed electron level [6].

Model. - The charging Hamiltonian has the form

$$
\hat{H}_{c}=E_{C}\left(\hat{N}-N_{0}\right)^{2}, \quad \hat{N}=\sum_{\alpha} \int_{V} \hat{\psi}_{\alpha}^{\dagger}(\mathbf{r}) \hat{\psi}_{\alpha}(\mathbf{r}) d^{3} r
$$

the sum being taken over the spin indices $\alpha$ and the integration domain is the volume $V$ of the island. Here $N_{0}$ is the background charge supplied by ions and by the external circuit. The kinetic energy has the usual form,

$$
H_{\text {kin }}=\sum_{\alpha} \int d^{3} r \hat{\psi}_{\alpha}^{\dagger}(\mathbf{r})\left[-\frac{\hbar^{2} \nabla^{2}}{2 m}-E_{F}+V(\mathbf{r})\right] \hat{\psi}_{\alpha}(\mathbf{r}),
$$

where $V(\mathbf{r})=I[\delta(x+d / 2)+\delta(x-d / 2)]$ describes the insulating barrier between the island and the leads, $x$ is the coordinate along the conductor. 
Superconductivity in the leads is described by the usual BCS Hamiltonian where $\Delta(\mathbf{r})=|\Delta| e^{i \chi_{L, R}}$ inside the leads, i.e., for $x<-d / 2$ or $x>d / 2$, respectively, while $\Delta=0$ in the normal conductor, $-d / 2<x<d / 2$.

In this model, the states of the system are spinindependent. The advanced and retarded Green functions in the real-frequency representation can be found from the Dyson equation

$$
\int d^{3} r \check{L}\left(\mathbf{r}_{1}, \mathbf{r}\right) \check{G}_{\epsilon}^{R(A)}\left(\mathbf{r}, \mathbf{r}_{2}\right)=\delta\left(\mathbf{r}_{1}-\mathbf{r}_{2}\right)
$$

where

$$
\begin{aligned}
& \check{L}\left(\mathbf{r}_{1}, \mathbf{r}\right)=\check{G}_{0}^{-1}\left(\mathbf{r}_{1}\right) \delta\left(\mathbf{r}-\mathbf{r}_{1}\right)+2 E_{C} h\left(\mathbf{r}_{1}\right) \check{K}\left(\mathbf{r}_{1}, \mathbf{r}\right), \\
& \check{G}_{0}^{-1}(\epsilon, \mathbf{r})=\left(\begin{array}{cc}
-\epsilon+\hat{H}_{0} & -\Delta \\
\Delta^{*} & \epsilon+\hat{H}_{0}
\end{array}\right), \check{G}=\left(\begin{array}{cc}
G & F \\
-F^{\dagger} & \bar{G}
\end{array}\right),
\end{aligned}
$$

are matrixes in the Nambu space, and

$$
\begin{aligned}
& \hat{H}_{0}=-\hbar^{2} \nabla^{2} / 2 m-E_{F}+V(\mathbf{r})+U_{C} h(\mathbf{r}), \\
& U_{C}=2 E_{C}\left(N-N_{0}\right), \quad N=-\int_{V} d^{3} r \operatorname{Tr} \check{K}(\mathbf{r}, \mathbf{r}) .
\end{aligned}
$$

The function $h(\mathbf{r})=1$ if $\mathbf{r}$ belongs to the island and $h(\mathbf{r})=0$ otherwise. The kernel

$$
\check{K}\left(\mathbf{r}_{1}, \mathbf{r}\right)=\int \frac{d \epsilon}{4 \pi i} \check{G}_{\epsilon}^{K}\left(\mathbf{r}_{1}, \mathbf{r}\right)
$$

is determined by the Keldysh function, $\check{G}_{\epsilon}^{K}\left(\mathbf{r}_{1}, \mathbf{r}_{2}\right)=$ $\left[\check{G}_{\epsilon}^{R}\left(\mathbf{r}_{1}, \mathbf{r}_{2}\right)-\breve{G}_{\epsilon}^{A}\left(\mathbf{r}_{1}, \mathbf{r}_{2}\right)\right] f_{1}(\epsilon)$. Here $f_{1}(\epsilon)=1-2 n_{\epsilon}$ and $n_{\epsilon}$ is the occupation number. In equilibrium $n_{\epsilon}$ is the Fermi function, and $f_{1}(\epsilon)=\tanh (\epsilon / 2 T)$. The energy $U_{C}$ describes the variation of the charge on the island due to the change in the number of excitations while $N$ has a meaning of the excitation charge on the island.

Bogoliubov-de Gennes (BdG) equations. - Let us expand the retarded and advanced Green functions in a set of orthogonal and normalized functions as

$$
\check{G}_{\epsilon}^{R(A)}\left(\mathbf{r}_{1}, \mathbf{r}_{2}\right)=\sum_{n} \frac{\check{U}_{n}\left(\mathbf{r}_{1}\right) \check{U}_{n}^{\dagger}\left(\mathbf{r}_{2}\right)}{E_{n}-\epsilon \mp i \delta}, \check{U}_{n}(\mathbf{r})=\left(\begin{array}{r}
u_{n}(\mathbf{r}) \\
-v_{n}(\mathbf{r})
\end{array}\right),
$$

$\check{U}_{n}^{\dagger}=\left(u_{n}^{*}, v_{n}^{*}\right)$. The functions $u$ and $v$ satisfy the linear equations with effective potentials determined by $K_{u u}\left(\mathbf{r}, \mathbf{r}_{1}\right)$ and $K_{u v}\left(\mathbf{r}, \mathbf{r}_{1}\right)$ :

$$
\begin{aligned}
& \hat{H}_{0} u_{n}(\mathbf{r})+\Delta(\mathbf{r}) v_{n}(\mathbf{r})+E_{C} h(\mathbf{r}) \int_{V} K_{u u}\left(\mathbf{r}, \mathbf{r}_{1}\right) u_{n}\left(\mathbf{r}_{1}\right) d^{3} r_{1} \\
& -E_{C} h(\mathbf{r}) \int_{V} K_{u v}\left(\mathbf{r}, \mathbf{r}_{1}\right) v_{n}\left(\mathbf{r}_{1}\right) d^{3} r_{1}=E_{n} u_{n}(\mathbf{r}) \\
& \hat{H}_{0} v_{n}(\mathbf{r})-\Delta^{*}(\mathbf{r}) u_{n}(\mathbf{r})+E_{C} h(\mathbf{r}) \int_{V} K_{u u}^{*}\left(\mathbf{r}, \mathbf{r}_{1}\right) v_{n}\left(\mathbf{r}_{1}\right) d^{3} r_{1} \\
& \quad+E_{C} h(\mathbf{r}) \int_{V} K_{u v}^{*}\left(\mathbf{r}, \mathbf{r}_{1}\right) u_{n}\left(\mathbf{r}_{1}\right) d^{3} r_{1}=-E_{n} v_{n}(\mathbf{r})
\end{aligned}
$$

The kernels $K_{u u}\left(\mathbf{r}, \mathbf{r}_{1}\right)$ and $K_{u v}\left(\mathbf{r}, \mathbf{r}_{1}\right)$ are determined self-consistently. They can also be expanded into the same wave functions,

$$
\begin{aligned}
K_{u u}\left(\mathbf{r}, \mathbf{r}_{1}\right) & =\sum_{m} u_{m}(\mathbf{r}) u_{m}^{*}\left(\mathbf{r}_{1}\right) f_{1}\left(E_{m}\right) \\
K_{u v}\left(\mathbf{r}, \mathbf{r}_{1}\right) & =\sum_{m} u_{m}(\mathbf{r}) v_{m}^{*}\left(\mathbf{r}_{1}\right) f_{1}\left(E_{m}\right) .
\end{aligned}
$$

The kernels obey the symmetry $K_{u u}\left(\mathbf{r}, \mathbf{r}_{1}\right)=$ $-K_{v v}^{*}\left(\mathbf{r}, \mathbf{r}_{1}\right)=K_{u u}^{*}\left(\mathbf{r}_{1}, \mathbf{r}\right)$ and $K_{u v}\left(\mathbf{r}, \mathbf{r}_{1}\right)=K_{v u}^{*}\left(\mathbf{r}, \mathbf{r}_{1}\right)=$ $K_{u v}\left(\mathbf{r}_{1}, \mathbf{r}\right)$ due to the symmetry of the functions $u_{E} \rightarrow v_{-E}^{*}, v_{E} \rightarrow-u_{-E}^{*}$ with respect to $E \rightarrow-E$. The excitation charge is also expressed through $u_{n}$ and $v_{n}$, $N=\frac{1}{2} \sum_{n} N_{n}$, where

$$
N_{n}=-\int d V\left[u_{n}^{*}(\mathbf{r}) u_{n}(\mathbf{r})-v_{n}^{*}(\mathbf{r}) v_{n}(\mathbf{r})\right] f_{1}\left(E_{n}\right)
$$

is the number of excitations on the state $n$, it determines the average excitation charge $Q_{n}=e N_{n}$ carried by the state. The sum runs over the states with both positive and negative energies. It can be written as a sum over only positive-energy states: $N=\sum_{n, E_{n}>0} N_{n}$.

Andreev states. - The normal conductor has a single transport mode parameterized by the coordinate $x$ along it. The eigenstates in a short low-transparency junction have the form of narrow particle-like and hole-like resonances such that only two of them fit into the sub-gap range of $|\Delta|$. Due to proximity to the superconducting leads these states transform into the Andreev states localized over the distances of the order of $\xi$ near the junction. All other resonances are essentially the same as in the normal state; thus they can be ignored if one is interested in energies of the order of the superconducting gap. The two Andreev states will be labeled with $n=+1$ and $n=-1$. One of them has a positive and other has a negative energy $E_{1}=-E_{-1}>0$. In what follows we assume that the transparency is so small that $\mathcal{T} \ll d / \xi$. In this limit the Andreev states are mostly concentrated inside the normal conductor. We will also consider energies much smaller that the superconducting gap because it is this energy range where the supercurrent enhancement is most pronounced. In this case the kernels Eqs. (6) in Eqs. (4) and (5) contain contributions only from the two Andreev states, $n= \pm 1$. Due to the symmetry with respect to $E \rightarrow-E$,

$$
\begin{aligned}
K_{u u}\left(x, x_{1}\right) & =\left[u_{1}(x) u_{1}^{*}\left(x_{1}\right)-v_{1}^{*}(x) v_{1}\left(x_{1}\right)\right] f_{1}\left(E_{1}\right), \\
K_{u v}\left(x, x_{1}\right) & =\left[u_{1}(x) v_{1}^{*}\left(x_{1}\right)+v_{1}^{*}(x) u_{1}\left(x_{1}\right)\right] f_{1}\left(E_{1}\right) .
\end{aligned}
$$

Denoting $u_{1}=u^{+} e^{i p_{u} x}+u^{-} e^{-i p_{u} x}$ and $v_{1}=v^{+} e^{i p_{v} x}+$ $v^{-} e^{-i p_{v} x}$ and neglecting the difference between the momenta of $u$ and $v$ in the kernels, $p_{u}=p_{v} \equiv p$ (for a short 
junction, $\left.p_{u}-p_{v} \sim E / v_{F} \ll 1 / d\right)$ we find

$$
\begin{gathered}
\int_{-d / 2}^{d / 2} d x_{1}\left[K_{u u}\left(x, x_{1}\right) u_{1}\left(x_{1}\right)-K_{u v}\left(x, x_{1}\right) v_{1}\left(x_{1}\right)\right] \\
=-u_{1}(x) N_{1}-v_{1}^{*}(x) M_{1} \\
\int_{-d / 2}^{d / 2} d x_{1}\left[K_{u u}^{*}\left(x, x_{1}\right) v_{1}\left(x_{1}\right)+K_{u v}^{*}\left(x, x_{1}\right) u_{1}\left(x_{1}\right)\right] \\
=-v_{1}(x) N_{1}+u_{1}^{*}(x) M_{1}
\end{gathered}
$$

$N_{1}$ being the number of excitations on the state $n=1$ while $M_{1}=2 \int_{-d / 2}^{d / 2} u_{1}\left(x_{1}\right) v_{1}\left(x_{1}\right) f_{1}\left(E_{1}\right) d x_{1}$.

The BdG equations (4), (5) inside the normal conductor take the form

$$
\begin{aligned}
& \left(-\frac{\hbar^{2}}{2 m} \frac{d^{2}}{d x^{2}}-\mu\right) u_{1}-E_{C} M_{1} v_{1}^{*}=E_{1} u_{1} \\
& \left(-\frac{\hbar^{2}}{2 m} \frac{d^{2}}{d x^{2}}-\mu\right) v_{1}+E_{C} M_{1} u_{1}^{*}=-E_{1} v_{1}
\end{aligned}
$$

where the effective chemical potential is

$$
\mu=E_{F}-U_{C}+E_{C} N_{1}
$$

For $u, v^{*} \propto e^{i p x}$ we find from here

$$
p^{2} / 2 m-\mu= \pm \sqrt{E_{1}^{2}-E_{g}^{2}}
$$

where $E_{g}=E_{C}\left|M_{1}\right|$ is the Coulomb gap. Using the solutions of the BdG equations without the Coulomb interaction, one can show that $M_{1} \sim \mathcal{T}$. The Andreev state energy is (see below) $E_{1} \gtrsim\left(\hbar v_{F} / d\right) \mathcal{T}$. Therefore, the Coulomb gap $E_{g} \sim E_{C} \mathcal{T}$ in Eq. (9) can be neglected when $\gamma \ll 1$. In this limit Eq. (9) reduces to its usual form, $p^{2} / 2 m-\mu= \pm E_{1}$, while the functions $u$ and $v$ inside the normal conductor are decoupled.

The BdG equations for the entire system can thus be written in a standard form

$$
\begin{array}{r} 
\\
{\left[\hat{H}_{0}-h(\mathbf{r}) E_{C} N_{1}\right] u_{1}(\mathbf{r})+\Delta(\mathbf{r}) v_{1}(\mathbf{r})=E_{1} u_{1}(\mathbf{r}),} \\
-\left[\hat{H}_{0}-h(\mathbf{r}) E_{C} N_{1}\right] v_{1}(\mathbf{r})+\Delta^{*}(\mathbf{r}) u_{1}(\mathbf{r})=E_{1} v_{1}(\mathbf{r}) .
\end{array}
$$

The BdG equations (10), (11) determine the energy of the Andreev bound states as a function of the phase difference $\phi=\chi_{R}-\chi_{L}$. For low transparency, $\mathcal{T} \ll d / \xi$, the energies $E_{ \pm 1}= \pm\left(\hbar v_{F} / d\right) \epsilon_{A}$ in the range $E \ll|\Delta|$ have the form [3, 8]

$$
\epsilon_{A}=\sqrt{z^{2}+\mathcal{T}^{2} \cos ^{2}(\phi / 2)}
$$

where $\mathcal{T}=\mathcal{T}_{1} / 2$ is the transparency of two contacts in series. The quantity $z$ measures the shift of the chemical potential $\mu$ inside the conductor, Eq. (8), from one of the resonances. It is defined through the equation

$$
p_{F} d / \hbar+\eta+\left(d / \hbar v_{F}\right)\left[E_{C} N_{1}-U_{C}\right]=\pi k+z
$$

where $\eta=\arctan \sqrt{\mathcal{T}_{1}}$ is the scattering phase shift and $k$ is an integer. The range of applicability of Eq. (12) is $\epsilon_{A} \ll d / \xi$.

As it follows from Eq. (77), $U_{C}=U_{C}^{\prime}+2 E_{C} N_{1}$ where $U_{C}^{\prime}=2 E_{C}\left(N^{\prime}-N_{0}\right)$ and $N^{\prime}=\sum_{n \neq 1} N_{n}$. Let us denote

$$
y=p_{F} d / \hbar+\eta-d U_{C}^{\prime} / \hbar v_{F}-\pi k .
$$

The quantity $y$ depends on the gate voltage through the background charge $N_{0}$. The excitation number $N^{\prime}$ changes little if the chemical potential varies within one resonance, $z \lesssim 1$. Therefore, the chemical potential shift $y$ can be considered as a parameter controlled by the gate voltage.

For a localized state [8]

$$
N_{1}=\left(\frac{\partial E_{1}}{\partial \mu}\right)_{\phi} f_{1}\left(E_{1}\right)
$$

The subscript $\phi$ means that the phase is kept constant. As it follows from Eq. (8) for the effective chemical potential of the Andreev state, $\partial \mu / \partial z=\hbar v_{F} / d$. Therefore, Eq. (13) yields

$$
y=z+\gamma \frac{\partial \epsilon_{A}}{\partial z} f_{1}\left(E_{1}\right) .
$$

We consider zero temperatures and put $f_{1}\left(E_{1}\right)=1$. This can be done if temperature satisfies $T \ll \mathcal{T} \delta E \sim$ $\Delta \mathcal{T}(\xi / d)$. Equation (15) is the central result of this paper. Equations (12) and (15) determine $\epsilon_{A}$ as a function of $y$ and Eq. (14) then determines the charge $N_{1}$ on the Andreev level.

Results. - Equations (12) and (15) yield

$$
y=\left(1+\gamma / \epsilon_{A}\right) \sqrt{\epsilon_{A}^{2}-\mathcal{T}^{2} \cos ^{2}(\phi / 2)} \text {. }
$$

We see that at $\gamma \neq 0$ ( i.e., due to the Coulomb interaction) there appears a substantial region of gate voltages, $y \lesssim \gamma$, where energy is close to its smallest possible value, $\mathcal{T} \cos (\phi / 2)$. This is more pronounced if the Coulomb energy is larger than the gap $E_{C} /|\Delta| \gg 1$ (estimates will be given later). Indeed, since $\epsilon_{A} \lesssim d / \xi$ the ratio $\gamma / \epsilon_{A}>E_{C} /|\Delta|$. Yet more drastic effect is expected if $\mathcal{T} \ll \gamma$. In this case the term $\gamma / \epsilon_{A}$ in brackets in Eq. (16) dominates. As a result, for $y<\gamma$

$$
\epsilon_{A}=\frac{\mathcal{T} \cos (\phi / 2)}{\sqrt{1-y^{2} / \gamma^{2}}} .
$$

According to Eqs. (16) or (17), $\epsilon_{A}$ and $z$ remain small $\epsilon_{A}, z \ll \gamma$ in the range of gate voltages $y \lesssim \gamma$. Thus the energy satisfies $E_{1} \ll E_{C},|\Delta|$, and the Kondo regime is not realized.

Figure1 (upper panels) shows the solutions of Eqs. (16) for $\mathcal{T}=0.1$ and for two values of the Coulomb interaction, $\gamma=0.2$ (left panel) and $\gamma=0$ (right panel). One 
observes that $\epsilon_{A} \rightarrow 0$ as $\phi$ approaches $\pi$ if $y<\gamma$. The decrease in energy is, of course, cut off when $|\phi-\pi| \sim$ $(T /|\Delta|)(d / \xi \mathcal{T})$ because of the decreasing factor $f_{1}\left(E_{1}\right)$ in Eq. (15). This energy behavior has a crucial impact on the supercurrent given by the expression [7]:

$$
j_{s}=-\frac{2 e}{\hbar}\left(\frac{\partial E_{1}}{\partial \phi}\right)_{\mu} f_{1}\left(E_{1}\right)=j_{0} \frac{\mathcal{T} \sin \phi}{\epsilon_{A}}
$$

where $j_{0}=e v_{F} \mathcal{T} / 2 d$. Since the energy remains small in a wide range of gate voltages, the supercurrent is essentially enhanced as compared to its zero-Coulomb value for the same gate voltage. In particular, for comparatively large Coulomb interaction, $\gamma \gg \mathcal{T}$, we have from Eq. (17)

$$
j / j_{0}=2 \sqrt{1-y^{2} / \gamma^{2}} \sin (\phi / 2) .
$$

The critical current $j_{c}=2 j_{0} \sqrt{1-y^{2} / \gamma^{2}}$ is reached at $\phi=\pi$. It is roughly by factor $\gamma / \mathcal{T}$ larger than its value without the Coulomb interaction, $j_{c}=j_{0} \mathcal{T} / y$, reached at $\phi=\pi / 2$ for the same gate voltage $y \gg \mathcal{T}$. The supercurrent Eq. (18) is shown in Fig. 1 (lower panels) for $\mathcal{T}=0.1$ and for two value of the Coulomb interaction, $\gamma=0.2$ (left panel) and $\gamma=0$ (right panel). One observes significant enhancement of the supercurrent for a given gate voltage.

Discussion. - We have shown that charging of the Andreev level leads to a strong enhancement of the supercurrent in a wide range of gate voltages (parameterized by the quantity $y$ ). The charge of the Andreev state compensates the deviation of the chemical potential caused by the change in the gate voltage thus preserving the resonance transmission of the double barrier structure and increasing the transparency of the device.

The Coulomb interaction is characterized by the dimensionless parameter $\gamma=d E_{C} / \hbar v_{F}$. The upper estimate gives $\gamma \sim e^{2} / \hbar v_{F} \lesssim 1$. Our approximation requires small $\gamma$. Nevertheless, the factor $\gamma / \mathcal{T}$ that determines the enhancement of the supercurrent can reach quite large values due to low transparency. A substantial enhancement of supercurrent occurs already when $E_{C} \gtrsim|\Delta|$. A typical gap $\Delta$ (as for $\mathrm{Al}$ ) is about $1 \mathrm{~K}$. The Coulomb energy $E_{C}=e^{2} / 2 C$ of $1 \mathrm{~K}$ corresponds to the capacitance $C \approx 8 \cdot 10^{-4} \mathrm{~cm}$. Therefore, to have $E_{C} \gg \Delta$ the characteristic size of the normal conductor should be smaller than $\sim 10^{-3} \mathrm{~cm}$ which can be easily realized in practice.

We thank I. Sadovskyy, V. Shumeiko, and A. Zazunov for stimulating discussions. This work was supported by the Russian Foundation for Basic Research under grant 09-02-00573-a, by the Program "Quantum Physics of Condensed Matter" of the Russian Academy of Sciences, by the Academy of Finland Centers of Excellence Program, by the U.S. Department of Energy Office of Science under Contract No. DE-AC02-06CH11357, and by Norwegian Research Council through the program on sensors and detectors.
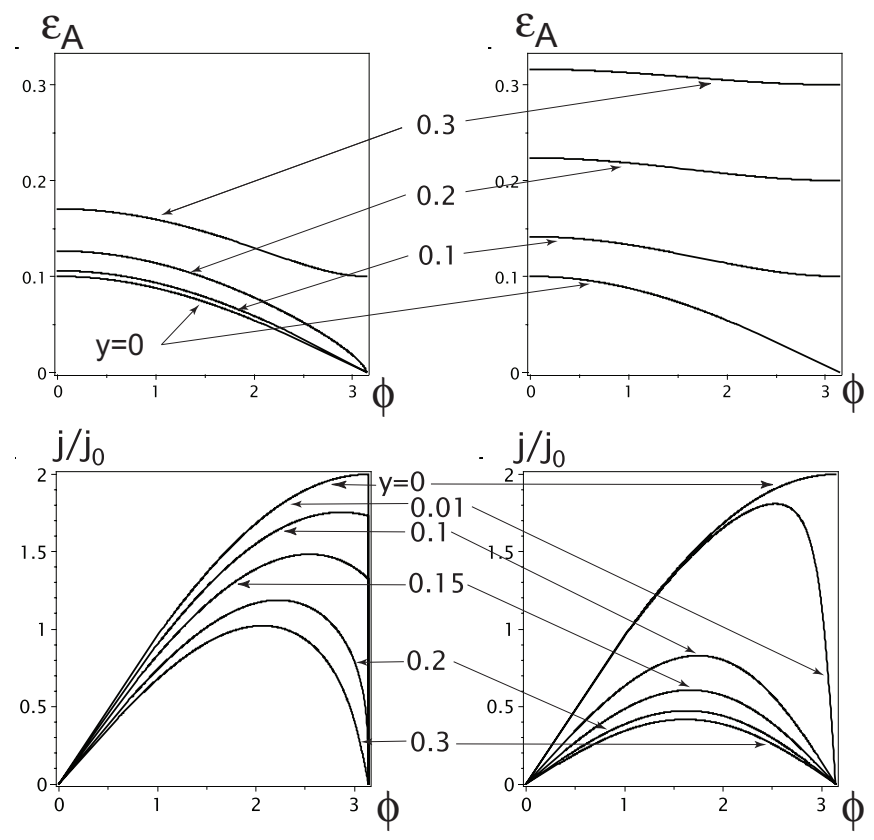

FIG. 1. Andreev state energy (upper panels) and the supercurrent (lower panels) in the presence of Coulomb interaction, $\gamma=0.2$ (left panels) and without it, $\gamma=0$ (right panels) as functions of the phase difference $\phi$ and the gate voltage $y$ for $\mathcal{T}=0.1$. The curves for $\gamma=0$ and $\gamma=0$ coincide at $y \rightarrow 0$. The curves for the supercurrent at $\gamma=0.2$ for $y=0$ and $y=0.01$ overlap, while at $\gamma=0$ they significantly differ at $\phi$ close to $\pi$.

[1] K. K. Likharev, IBM J. Res. Dev. 32, 144 (1988); see for a review D. V. Averin and K. K. Likharev, in Mesoscopic Phenomena in Solids, ed. by B. L. Altshuler, P. A. Lee, and R. A. Webb (Elsevier, Amsterdam, 1991); Yu. V. Nazarov and Ya. M. Blanter, Quantum transport (Cambridge University Press, 2009).

[2] M. Bockrath et al., Nature (London) 397, 598 (1999); A. Yu. Kasumov et al., Science 284, 1598 (1999); Z. Yao et al., Nature (London) 402, 273 (1999); M. R. Buitelaar, T. Nussbaumer, and C. Schönenberger, Phys. Rev. Lett.89, 256801 (2002); P. Jarillo-Herrero, J. A. van Dam, and L. P. Kouwenhoven, Nature 439, 953 (2006); H. I. Jorgensen et al., Phys. Rev. Lett.96, 207003 (2006); A. Eichler et al., Phys. Rev. B79, 161407(R) (2009).

[3] I. A. Sadovskyy, G. B. Lesovik, and G. Blatter, Phys. Rev. Lett. 75, 195334 (2007); JETP Letters 86, 210 (2007).

[4] F. Dolcini et al., Phys. Rev. Lett.91, 266402 (2003).

[5] N. B. Kopnin, Y. M. Galperin, and V. M. Vinokur, Phys. Rev. B 79, 035319 (2009).

[6] L. I. Glazman and K. A. Matveev, JETP Lett. 49, 659 (1989); A. V. Rozhkov and D. P. Arovas, Phys. Rev. Phys. Rev. Lett.82, 2788 (1999); A. A. Clerk and V. Ambegaokar, Phys. Rev. B61, 9109 (2000); A. V. Rozhkov and D. P. Arovas, ibid. 62, 6687 (2000); F. Siano and R. Egger, Phys. Rev. Lett.93, 047002 (2004); M. S. Choi et al., Phys. Rev. B70, 020502(R) (2004); G. Sellier et al., ibid. 72, 174502 (2005); C. Karrasch, A. Oguri, 
and V. Meden, ibid. 77, 024517 (2008); M. Governale, M. G. Pala, and J. König, ibid. 77, 134513 (2008); A. Zazunov, A. Schulz, and R. Egger, Phys. Rev. Lett.102, 047002 (2009); T. Meng, S. Florens, and P. Simon, Phys. Rev. B79, 224521 (2009); E. Vecino, A. Martín-Rodero, and A. Levy Yeyati, Phys. Rev. B 68, 035105 (2003);
A. Zazunov, A. Levy Yeyati, and R. Egger, Phys. Rev. B81 012502 (2010).

[7] C. W. J. Beenakker, Phys. Rev. B, 44, 1646 (1991).

[8] N. B. Kopnin, A. S. Melnikov, and V. M. Vinokur, Phys. Rev. Lett. 96, 146802 (2006). 